\title{
Internet videos and colorectal cancer in mainland China: a content analysis
}

\author{
Shun Zhang ${ }^{*}$, Yao Yang, Dongyi Yan, Biao Yuan, Xiaohua Jiang ${ }^{*}$ and Chun Song ${ }^{*}$ (D
}

\begin{abstract}
Background: Colorectal cancer incidence and mortality have been increasing in China and as one of the most important health problems facing the nation. Adequate dissemination of correct information about colorectal cancer could help in reducing cancer-related morbidity and mortality. This study aims to assess the completeness and reliability of colorectal cancer-related information available on the video website of Youku in mainland China.

Methods: Youku (https://www.youku.com/) was searched on September 15, 2016 for the search terms colorectal cancer. Only Chinese videos were included. Two reviewers independently evaluate the videos for characteristics, information source and usefulness. Content was analysed under six categories (aetiology, anatomy, symptoms, preventions, treatments and prognosis). Completeness was evaluated with a checklist developed by the researchers. Any discrepancies were resolved by consensuses. SPSS software was used to analyze data.

Results: There were 242 videos with relevant information about colorectal cancer. The type of source were as follows: independent users, 118 (49\%); health information web sites, 60 (25\%); medical doctors, 31 (13\%); news network, 22 (9\%); and hospital/university, 11 (4\%). In all, 57\% of videos had useful information about colorectal cancer, $21 \%$ were misleading. Videos posted by medical doctors $(P=0.021)$ and health information web sites $(p=0.039)$ were less incomplete than videos by independent users. Of the Traditional Chinese medicine (TCM) videos, 97 (76\%) had information about treatments of colorectal cancer. 30\% TCM videos contain misleading information, whose misleading rate was higher than total's (21\%).
\end{abstract}

Conclusions: The colorectal cancer videos in mainland China represented by Youku varied base on ownership and content and information incompleteness were fairly high. It is necessary that professionals adapt to the advanced technology and think useful methods to solve the variable quality of information of internet video websites in mainland China.

Keywords: Colorectal cancer, Internet, Youku, Mainland China

\section{Background}

Cancer incidence and mortality have been increasing in China and have created a significant number of health concerns [1]. Colorectal cancer ranks the fifth most commonly diagnosed cancer among male and female in China [2]. The ratio of estimated new colorectal cancer mortality incidence is $50.8 \%$ in China for 2015 [2] compared with 36.3\% in the United States for 2016 [3]. This considerably higher ratio means cancer prevention and control in China lags behind some Western countries.

\footnotetext{
*Correspondence: v2zs@hotmail.com; jiangxiaohua@163.com; chunsong163@163.com

Department of Gastrointestinal Surgery, Shanghai East Hospital (East Hospital Affiliated to Tongji University), 150 Jimo Road, Shanghai 200120, People's Republic of China
}

Up to 31 December 2016, it was reported that 731 million Chinese internet users, and more than 695 million people were using mobile devices to quick browse online information. Over 570 million online video users accounted for three-quarters of total internet users [4]. Health and medical treatment has been the most popular science topics in mainland China [5]. Freely available video websites, such as YouTube, are popular sources of information dissemination with more than 100 million viewers every day [6]; however, YouTube is blocked in China because of Chinese internet censorship.

Chinese people prefer online video websites, such as Yoku, iQiyi, Sohu Tv or Tencent Video. Youku is the most popular source of video blogs and short original 
videos uploaded by individuals in mainland China [7]. Youku initially emphasized user-generated content. The average number of daily video views was 1.18 billion [8]. The number of monthly active users was over 500 million, and $60 \%$ of audiences were male [7]. Youku features the same kinds of videos on YouTube and is considered the largest Chinese video broadcast site. Similar to YouTube, the posted videos are not peer control, could be uploaded from different sources and are likely to be of variable quality [9].

Many studies reported that video broadcast sites have positive and negative effects on health information dissemination. Some videos can provide useful resources for knowledge and were used by medical students as a learning resource $[10,11]$. Videos may promote misleading information, such as disparaging vaccinations [9] and describing ineffective or potentially dangerous natural therapies for gallstone disease [12]. Not only were audience attempting therapies that may be harmful, but they were not going in for accurate therapy, which can lead to other complications.

The use of video broadcasting sites as a source of information in disease areas, especially in colorectal cancer in mainland China, has not been evaluated. Thus, the present study aimed at evaluating the completeness and reliability of Chinese-language colorectal cancer-related information available on the video website of Youku in mainland China; assess the overall quality of online information on colorectal cancer; and share our thoughts on important future directions for managing information about colorectal cancer on websites of mainland China.

\section{Method}

We searched Youku (www.youku.com) on November 15, 2016 to locate video clips containing relevant information about colorectal cancer in human patients. The keyword "colorectal cancer" was used to identify related video clips. Videos that were duplicated, not in Chinese and not directly related to the investigated condition were excluded.

We included all unique videos with Chinese language content that contained any message about human colorectal cancer. All videos were downloaded and saved. We assessed each video according to the following characteristics: duration, ownership, number of views, video quality, and colorectal cancer content. Ownership was classified by medical doctor, hospital/university, news network, health information website or independent user.

All videos were viewed and analysed for content by 2 reviewers, and disagreements were resolved by an arbitrator. All researchers had medical background and specialized in gastrointestinal surgery. All researchers had finished their respective residencies at general hospital and had enough experience in the diagnosis and management of colorectal cancer. The reviewers classified the videos as useful, misleading or useless, as defined by the following: useful-containing scientifically correct information about any aspect of the disease: symptoms, treatment, and prevention; misleading - containing scientifically unproven information; useless-without containing the any aspect of colorectal cancer or addressing personal experience. If the video included trustable and misleading information at the same time, the videos were categorized as "misleading".

We assessed the quality of each video using a completeness score (Table 1). Two reviewers viewed each video in all content areas (aetiology, anatomy, symptoms, preventions, treatments and prognosis). At present, no validated tool for this purpose exists in the literature. Any disagreements were resolved with consensuses.

Traditional Chinese medicine (TCM) has been is deeply embedded in the populations of China and applied to the prevention and treatment of various diseases from ancient times until now. TCM is promoted and institutionalized by the Chinese government, has spread to more than 100 countries and has grown into an international industry [13]. For this reason, we also analysed TCM content regarding colorectal cancer in our study. Inter-observer agreement was evaluated with a kappa coefficient. Differences between groups were compared with a one-way ANOVA. Data analysis was performed with SPSS Version 16 Software. If the $p$-value is less than 0.05 , the result was considered to be significant.

Table 1 Completeness checklist

\begin{tabular}{ll}
\hline Content & Description \\
\hline Aetiology & Precancerous lesion \\
& Heredity \\
& Eating habits \\
Anatomy & - \\
Symptoms & Stool change \\
& Altered bowel habits \\
& Abdominal pain \\
& Abdominal mass \\
& Systemic symptoms \\
Preventions & Screening \\
Treatments & Daily habits \\
& Surgery \\
& Chemotherapy \\
& Radiotherapy \\
Prognosis & Traditional Chinese medicine \\
& TNM stage \\
& Perioperative treatments \\
& Others \\
\hline
\end{tabular}




\section{Result}

A Youku search revealed 348 videos for colorectal cancer. Videos were removed for a variety of reasons (Table 2). Video duplication and not being in Chinese were the two main reasons. Of the 348 videos screened, 242 videos met the inclusion criteria.

\section{Ownership}

A total of $49 \%$ of the videos were posted on the website by independent users. Health information websites were responsible for uploading $25 \%$ of the total videos. The videos contributed by medical doctors were only $13 \%$ but higher than other owner videos by max viewership and mean viewership (Table 3 ). This difference among groups was statistically significant $(p<0.05)$.

\section{Information reliability}

The 242 included videos were classified as useful (136 [57\%]), misleading (51 [21\%]), and useless (55 [22\%]) according to medical content (Table 4). The kappa coefficient statistics of agreement of these videos was 0.88 .

The number of videos containing misleading information was 51 . A large part (41 [80\%]) were amateur videos about personal experiences and emotions. The mean duration of the videos was $4.0 \mathrm{~min}$ with no significant differences between useful and misleading videos or between useful and irrelevant videos $(p<0.05)$.

\section{Content}

Useful videos were analysed based on the information they contained. In all of the categories, treatments were the most frequently covered topic (70\%), followed in descending order by symptoms (33\%), prognosis (26\%), anatomy (20\%), preventions (15\%) and aetiology (11\%). Table 5 shows the information completeness scores. Videos by medical doctors $(p=0.021)$ and health information websites $(p=0.039)$ sources were significantly more complete than those posted by independent users.

\section{Traditional Chinese medicine}

There were 128 videos containing TCM from diagnosis to treatment. Of the TCM videos, 97 (76\%) had information about treatments of colorectal cancer. Among these videos, 10 included TCM and Western medicine at the

Table 2 Reasons for excluding videos

\begin{tabular}{ll}
\hline Reason for exclusion & No. \\
\hline No audio & 1 \\
No video & 2 \\
Not in Chinese & 15 \\
Not related to subject & 3 \\
Duplicate & 85 \\
Total exclusions & 106 \\
\hline
\end{tabular}

same time. The information reliability is shown in Table 6. Medical doctors and university provided more reliable information than others $(p<0.05)$. A total of $30 \%$ TCM videos contain misleading information, and this misleading rate was higher than the totals (21\%). Among the videos containing both TCM and Western medicine, the misleading rate was as high as $90 \%$. Most of the videos exaggerated the actual effect of TCM and understated therapies, except for the health information websites mean viewership (798: 895). The other sources' mean viewership in TCM were higher than those containing both TCM and non-TCM videos.

\section{Discussion}

Colorectal cancer ranks as the fifth leading cause of cancer death among both male and female in mainland China. Because the population of China accounts for one fifth of the global world, colorectal cancer cases in China account for $22 \%$ of all newly diagnosed cases and $27 \%$ of all deaths from worldwide [14]. The effectiveness of prevention, early detection, and management of colorectal cancer is not only important for China but also for the world.

Internet video websites can provide useful diagnostic, treatment and preventative medical services information. Previous research has evaluated YouTube as an important source of information on disease topics [15]. Although YouTube is blocked due to many reasons in mainland China, there are many similar internet video websites delivering the same functionality, such as Youku. To the best of our knowledge, no study has been performed to assess the accuracy and usefulness of internet videos as a source of healthy information for colorectal cancer in mainland China.

In this study, we selected Youku.com as the target video website, which is ranked the largest Chinese video broadcast site. The website of Youku not only focuses on professionally produced videos but also emphasizes user-generated content. The monthly unique visitors of Youku were 2,6376,000,000 according to the data of October in 2016 [7].

Our study demonstrates approximately 242 videos addressing colorectal cancer were provided by different sources. Independents users represent the greatest number of sources. The content was mainly about personal experiences in surgical procedures or hospital stays. Our results also show that Chinese medical doctors and health related institutions comprising $17 \%$ of colorectal cancer videos do not pay sufficient attention to the platform for the distribution of information. Doctors in china frequently experience work overload, tend to work overtime and experience energy deficiencies, which seem to be one of reasons for this phenomenon [16]. The videos that were viewed most often were the videos posted 
Table 3 Sources and classification of detected videos

\begin{tabular}{|c|c|c|c|c|}
\hline Source & Total videos & Max viewership & Min viewership & Mean viewership \\
\hline Independent user & 118 & 53,455 & 8 & 10,621 \\
\hline Health information web site & 60 & 60,234 & 167 & 15,390 \\
\hline Medical doctor & 31 & 61,132 & 12,583 & 23,893 \\
\hline Hospital/University & 11 & 20,343 & 670 & 8783 \\
\hline News network & 22 & 57,890 & 4791 & 9321 \\
\hline
\end{tabular}

by doctors followed by health information websites. This indicates that people are more interested in a professionals experience regarding disease rather than their peers.

As the content of most videos often lacks peer or institutional quality review, many may not be subject to quality controls and may not be evidence-based; thus, it is not surprising that a majority of this content is misleading or irrelevant. According to previous studies, the dissemination of inaccurate information by video websites differs from diseases. A total of $56.5 \%$ of the video information on cholecystolithiasis [11], 16.2\% on H1N1 influenza [17] and $1.6 \%$ on acute appendicitis in children [18] on YouTube were misleading. In our study, it was demonstrated that only one-fifth of website videos contain no scientifically oriented information. Only 36\% of the independent users videos reviewed were considered to be useful compared with $90 \%$ useful doctors' videos.

The most commonly watched videos from independent users were those that contained misleading information, while the lowest number of views were from medical doctors and health information websites. These results also indicated that effective regulatory measures are needed to control scientifically accredited information. If misleading videos were less viewed by audiences, the harm might be reduced.

Regarding videos addressing colorectal cancer, it is highly difficult for laypeople or patients to distinguish between useful videos or those containing no accurate information. Our result indicates that an important element to assess the reliability of videos regarding colorectal cancer may be the ownership. If academic institutions represent the source, such as hospital/university or medical doctors, the videos may be regarded to be trustworthy on the basis of content [19]. The result is similar to those of other studies conducted outside of mainland China $[15,16]$.

We found that the average completeness scores were only $18 \%$ with a combination of aetiology, anatomy, symptoms, preventions, treatments and prognosis. Most of the included website videos only contained one of the above-mentioned categories. In all of the categories, treatments were the most frequently covered topic (70\%). It is unlikely to expect all videos to comprehensively cover all aspects of colorectal cancer; therefore, it should be deemed that some videos, whilst incomplete, do contain precise and valuable content. Our results indicated that videos from medical doctors and health related institutions have significantly higher completeness scores than those posted by independent users. This result may suggest that videos posted by layperson mainly aim a more social goal and videos posted by health and medical organizations commonly take a more educational purpose. The study indicated that professionals should utilize their expertise and contribute to more high-quality videos for patients as information sources in mainland China.

When video contents were analyzed, the most universal topic were the treatment aspects of the colorectal cancer. This finding may indicate that most publishers thought that treatment factors are the most important component of colorectal cancer. Surgery, chemotherapy and radiotherapy have been the mainstay of colorectal cancer treatment. Approximately $70 \%$ of videos contained one of the above subjects. As the country of origin and application of TCM, China has a unique TCM theoretical system and effective treatment methods. In mainland China, TCM has been recognized as additional

Table 4 Sources and classification of detected videos

\begin{tabular}{lllll}
\hline Ownership & Total Videos & Useful (Mv) & Misleading (Mv) & Useless (Mv) \\
\hline Independent users & 118 & $43(7689)$ & $41(18123)$ & $34(5283)$ \\
Health information web site & 60 & $48(17517)$ & $4(895)$ & $1(12583)$ \\
Medical doctors & 31 & $28(24588)$ & $0(-)$ & 0 \\
Hospital/University & 11 & $11(8783)$ & $6(4567)$ & $11(12583)$ \\
News network & 22 & $6(7302)$ & $4.3 \pm 2.1$ & $3.7 \pm 3.1$ \\
Mean duration (min \pm SD) & $4.0 \pm 2.3$ & $5.5 \pm 3.7$ & \\
\hline
\end{tabular}

Abbreviation: $S D$, standard deviation; Mean viewership: $M v$ 
Table 5 Completeness score

\begin{tabular}{lll}
\hline Completeness score & No & Mean \pm SD \\
\hline Aetiology & 15 & $1.53 \pm 0.51$ \\
Anatomy & 27 & - \\
Symptoms & 43 & $2.77 \pm 1.23$ \\
preventions & 21 & $1.24 \pm 0.44$ \\
Treatments & 95 & $2.07 \pm 0.83$ \\
Prognosis & 35 & $1.77 \pm 0.69$ \\
Total (max =17) & 136 & $3.07 \pm 1.94$ \\
\hline
\end{tabular}

treatment methods for colorectal cancer [20]. Our study shows that approximately 128 videos were about the anticancer properties of traditional Chinese medicine.

In oncology, TCM is believed to have great healing properties such as exerting specific anticancer activity or chemosensitisation to help in the individualization of anticancer treatment $[21,22]$. Chinese cancer patients frequently believe that herbs of TCM can help them against suffering from complications and to live well. Doctors trained in Western medicine published fewer videos than doctors trained in Chinese medicine. However, $30 \%$ of TCM videos contained misleading information that exaggerated actual effects and propaganda error messages, such as curing colorectal cancer. The highest total and misleading number of videos were posted by independent users. The meanest viewership was also from independent users. The misleading rate was higher than total misleading rate (21\%). There have been a large number of controlled clinical studies published in Chinese literature, but high-level evidence for the clinical efficacy of TCM is still lacking [23]. Mistakes were often found in professorial papers and in internet videos.

Colorectal cancer is characterized by high prevalence, a long asymptomatic period and eminently treatable precancerous lesions which, taken together, suggests that screening is a prudent option in mainland China [24]. For this reason, facilitating the earlier diagnosis of colorectal cancer may have a more immediate impact on the existing cancer burden in mainland China. A total of 21 videos contained colorectal cancer screening, which represented only $15 \%$ of all useful videos. Almost all screening videos address the importance of a Faecal Occult Blood Test, digital rectal exam, and colorectaloscopy.

Despite the rising colorectal cancer incidence, public awareness is still low in mainland China. Chinese internet websites, such as Youku, provide a different medium to disseminate colorectal cancer information to the public by video instead of written text. The written healthy information is commonly at a considerably higher reading level for Chinese patients. This video-based information source can help them and their caregivers get better understanding. Use of the internet for colorectal cancer information is likely to increase. It is necessary that professional individuals and academic institutions adapt to the advanced technology and think useful methods to solve the variable quality of information uploaded on internet video websites in mainland China. To maximize the potential of video-based information and minimize the quantity misleading or unhelpful information, multilateral efforts between doctors, governments and websites are needed.

\section{Limitations}

First, the main bias of our study was the subjectivity of judgement. There were no validated tools for assessing video data. Therefore, our classification method was subjective. However, the kappa statistic indicated quite high agreement between two reviewers. Second, there was no website, such as YouTube, with a clearly dominant position in China. Selecting only one Chinese video website's data may lead to some bias. Youku was the most popular website and had the largest audience in China. Youku in mainland China may still reflect the reliability of information available on video websites. Third, our results comprise a snapshot of information distribution to illustrate the quality of internet video at one point in time in China mainland, and these results may change according to the videos that can be added or removed with time.

Table 6 Treatments of Traditional Chinese medicine

\begin{tabular}{lllll}
\hline & Total videos & Useful (Mv) & Misleading (Mv) & Useless (Mv) \\
\hline Independent users & 62 & $23(13532)$ & $35(20021)$ & $4(6577)$ \\
Health information web site & 24 & $16(23122)$ & $3(798)$ & $5(1201)$ \\
Medical doctors & 7 & $7(27349)$ & $0(-)$ & $0(-)$ \\
Hospital/University & 1 & $1(13653)$ & $0(-)$ & $0(-)$ \\
News network & 3 & $1(12021)$ & $0(-)$ & $2(13216)$ \\
\hline
\end{tabular}

Abbreviation: Mean viewership: $M v$ 


\section{Conclusions}

Colorectal cancer videos represented by Youku in mainland China varied significantly by ownership and content and information incompleteness were fairly high. It is necessary that professionals adapt to the advanced technology and think useful methods to solve the variable quality of information uploaded on internet video websites in mainland China.

\section{Abbreviation}

TCM: Traditional Chinese medicine

\section{Acknowledgements}

None.

\section{Funding}

The study was supported by National Natural Scientific Foundation of China (No. 81700452) and in part by Japan China Sasakawa Medical Fellowship.

\section{Availability of data and materials}

The datasets used and/or analysed during this study are available from the corresponding author upon reasonable request. All the video were from Youku (www.youku.com) on November 15, 2016. Because everyday many new videos can be uploaded in the internet. Maybe now the number of videos have been changed in the website.

\section{Authors' contributions}

SZ, CS and XHJ developed the idea for the paper and led the development of the paper. SZ and YY conducted the data searches in Internet. SZ, DYY and BY extracted relevant data and analysis data. XHJ, CS and YY critically reviewed the manuscript for important intellectual content. SZ did the structure and writed the paper. All authors read and approved the final manuscript.

\section{Ethics approval and consent to participate}

This study did not require approval by the local Research Ethics Board as it involved publicly available data only.

\section{Consent for publication}

Not applicable.

\section{Competing interests}

The authors' declare that they have no competing interests.

\section{Publisher's Note}

Springer Nature remains neutral with regard to jurisdictional claims in published maps and institutional affiliations.

Received: 24 June 2018 Accepted: 22 November 2018

Published online: 04 December 2018

\section{References}

1. China NBoSo. China Statistical Yearbook 2016. Beijing: China Statistics Press; 2016.

2. Chen W, Zheng R, Baade PD, Zhang S, Zeng H, Bray F, Jemal A, Yu XQ, He J. Cancer statistics in China, 2015. CA Cancer J Clin. 2016;66:115-32.

3. Siegel RL, Miller KD, Fedewa SA, Ahnen DJ, Meester RG, Barzi A, Jemal A. Colorectal cancer statistics, 2017. CA Cancer J Clin. 2017;67(3):177-93.

4. China Internet Network Information Center Basic Data [(accessed on 18 June 2017)]. https://cnnic.com.cn/IDR/ReportDownloads/201706/ P020170608523740585924.pdf

5. China Science Communication Report on Chinese Netizens' Need and Search Behaviors of Science Communication, the Four Season [(accessed on 22 January 2017)] (In Chinese) http://index.baidu.com/special/kepu/.

6. Nason K, Donnelly A, Duncan HF. YouTube as a patient-information source for root canal treatment. Int Endod J. 2016;49:1194-200.
7. Chinese Web User Behavior Insight, iResearch PC index. [(accessed on 22 January 2017)] (In Chinese) http://index.iresearch.com.cn/pc/detail?id= $8173 \& \mathrm{kid}=78 \& \mathrm{Tid}=57$.

8. 20 Interesting Yoku Facts and Statistics [(accessed on 20 October 2018)] https://expandedramblings.com/index.php/youku-facts-statistics.

9. Keelan J, Pavri-Garcia V, Tomlinson G, Wilson K. YouTube as a source of information on immunization: a content analysis. JAMA. 2007;298:2482-4.

10. Azer SA, Algrain HA, AlKhelaif RA, AlEshaiwi SM. Evaluation of the educational value of YouTube videos about physical examination of the cardiovascular and respiratory system. J Med Internet Res. 2013;15:e24.

11. Azer SA, Aleshaiwi SM, Algrain HA, Alkhelaif RA. Nervous system examination on YouTube. BMC Med Educ. 2012;12:126.

12. Lee JS, Seo HS, Hong TH. YouTube as a source of patient information on gallstone disease. World J Gastroenterol. 2014;20:4066-70.

13. WHO. WHO Traditional Medicine Strategy: 2014-2023. Geneva: World Health Organization; 2013.

14. Ferlay JSI, Ervik M, Dikshit R, Eser SM. GLOBOCAN 2012 v1.0, Cancer incidence and mortality worldwide: IARC CancerBase no. 11, vol. 2013. Lyon: International Agency for Research on Cancer; 2017.

15. Hayanga AJ, Kaiser HE. Medical information on YouTube. JAMA. 2008;299: 1424-5 author reply 1425.

16. Wu H, Liu L, Wang Y, Gao F, Zhao X, Wang L. Factors associated with burnout among Chinese hospital doctors: a cross-sectional study. BMC Public Health. 2013:13:786.

17. Pandey A, Patni N, Singh M, Sood A, Singh G. YouTube as a source of information on the H1N1 influenza pandemic. Am J Prev Med. 2010;38:e1-3.

18. Adorisio O, Silveri M, De Peppo F, Ceriati E, Marchetti P, De Goyet Jde V. YouTube and pediatric surgery. What is the danger for parents? Eur J Pediatr Surg. 2015;25:203-5.

19. Syed-Abdul S, Fernandez-Luque L, Jian WS, Li YC, Crain S, Hsu MH, Wang YC, Khandregzen D, Chuluunbaatar E, Nguyen PA, Liou DM. Misleading health-related information promoted through video-based social media: anorexia on YouTube. J Med Internet Res. 2013:15:e30.

20. Goss PE, Strasser-Weippl K, Lee-Bychkovsky BL, Fan L, Li J, Chavarri-Guerra Y, Liedke PE, Pramesh CS, Badovinac-Crnjevic T, Sheikine Y, Chen Z, Qiao YL, Shao Z, Wu YL, Fan D, Chow LW, Wang J, Zhang Q, Yu S, Shen G, He J, Purushotham A, Sullivan R, Badwe R, Banavali SD, Nair R, Kumar L, Parikh P, Subramanian S, Chaturvedi P, lyer S, Shastri SS, Digumarti R, Soto-Perez-deCelis E, Adilbay D, Semiglazov V, Orlov S, Kaidarova D, Tsimafeyeu I, Tatishchev S, Danishevskiy KD, Hurlbert M, Vail C, St Louis J, Chan A. Challenges to effective cancer control in China, India, and Russia. Lancet Oncol. 2014;15:489-538.

21. Chiu J, Yau T, Epstein RJ. Complications of traditional Chinese/herbal medicines (TCM)--a guide for perplexed oncologists and other cancer caregivers. Support Care Cancer. 2009;17:231-40.

22. Ernst E. Complementary and alternative medicine (CAM) and cancer: the kind face of complementary medicine. Int J Surg. 2009;7:499-500.

23. Ma B, Guo J, Qi G, Li H, Peng J, Zhang Y, Ding Y, Yang K. Epidemiology, quality and reporting characteristics of systematic reviews of traditional Chinese medicine interventions published in Chinese journals. PLoS One. 2011;6:e20185

24. Huang W, Liu G, Zhang X, Fu W, Zheng S, Wu Q, Liu C, Liu Y, Cai S, Huang $Y$. Cost-effectiveness of colorectal cancer screening protocols in urban Chinese populations. PLoS One. 2014;9:e109150.

Ready to submit your research? Choose BMC and benefit from:

- fast, convenient online submission

- thorough peer review by experienced researchers in your field

- rapid publication on acceptance

- support for research data, including large and complex data types

- gold Open Access which fosters wider collaboration and increased citations

- maximum visibility for your research: over $100 \mathrm{M}$ website views per year

At $\mathrm{BMC}$, research is always in progress.

Learn more biomedcentral.com/submission 